\title{
An Exponential Strategy for Estimation of Population Mean in Systematic Sampling
}

\author{
Rajesh Tailor $^{1 *}$ and Asha Mishra ${ }^{2}$ \\ ${ }^{1}$ School of Studies in Statistics, Vikram University, Ujjain, M.P., India \\ ${ }^{1}$ School of Studies in Statistics, Vikram University, Ujjain, M.P., India \\ *Corresponding Author: ashadubey86@gmail.com
}

Available online at: www.isroset.org

Received: 29/Apr/2018, Revised: 10/May/2018, Accepted: 28/May/2018, Online: 30/Jun2018

\begin{abstract}
Using exponential function, an estimator for mean has been developed and it's Properties are studied. The mean squared error of the developed estimator has been derived. To compare developed estimator with existing estimators. An empirical comparative study is also conducted.
\end{abstract}

Keywords :- population mean, Exponential estimator ,Mean squared error.

\section{INTRODUCTION}

In systematic sampling, Shukla (1971) worked on classical ratio .An almost unbiased ratio and product estimators were envisaged by Singh and Singh (1998). Recently, Singh et al. (2011) studied ratio and product type exponential estimators in systematic sampling. In this paper a ratio-cum-product -type exponential estimator for population mean is suggested and it's properties are studied. For the present work, Let us consider a population $U=\left\{U_{1}, U_{2}, U_{3}, \ldots U_{N}\right\}$ of size $N$ serially numbered from 1 to $\mathrm{N}$. To draw a systematic sample, first unit is selected at random between 1 to $K$, where $K=\frac{\mathrm{N}}{\mathrm{n}}$. This unit is denoted by $i$ and then every $K^{\text {th }}$ unit is selected. This systematic sample is obtained as i, i+k, i+2k, i+3k ----- i+(n-1)k units.

Swain (1964) suggested ratio estimator for $\bar{Y}$ in systematic sampling as

$\hat{\overline{\mathrm{Y}}}_{\text {Rsys }}=\overline{\mathrm{y}}_{\mathrm{sys}}\left(\frac{\overline{\mathrm{X}}}{\overline{\mathrm{x}}_{\text {sys }}}\right)$

where $\bar{X}_{\text {sys }}=\frac{1}{n} \sum X_{i j}$ is an unbiased estimator of population mean $\bar{Y}$.

$\overline{\mathrm{X}}=\frac{1}{\mathrm{~N}} \sum_{\mathrm{j}=1}^{\mathrm{n}} \mathrm{X}_{\mathrm{ij}}$, the population mean of the auxiliary variate $\mathrm{x}$. Here, $\overline{\mathrm{X}}$ is assumed to be known. .

Shukla (1971) worked out product type estimator for population mean as

$\hat{\overline{\mathrm{Y}}}_{\text {Psys }}=\overline{\mathrm{y}}_{\mathrm{sys}}\left(\frac{\overline{\mathrm{z}}_{\text {sys }}}{\overline{\mathrm{Z}}}\right)$.

Using exponential function a ratio- type and a product- type estimator were worked out by Bahl and Tuteja (1991) as 
$\hat{\overline{\mathrm{Y}}}_{\mathrm{Re}}=\overline{\mathrm{y}} \times \exp \left(\frac{\overline{\mathrm{X}}-\overline{\mathrm{x}}}{\overline{\mathrm{X}}+\overline{\mathrm{x}}}\right)$

and

$\hat{\overline{\mathrm{Y}}}_{\mathrm{Pe}}=\overline{\mathrm{y}} \times \exp \left(\frac{\overline{\mathrm{z}}-\overline{\mathrm{Z}}}{\overline{\mathrm{Z}}+\overline{\mathrm{Z}}}\right)$.

$\hat{\bar{Y}}_{\text {Resys }}$ and $\hat{\bar{Y}}_{\text {Pesys }}$ was studied in systematic sampling by Singh et al. (2011) as

$\hat{\overline{\mathrm{Y}}}_{\text {Resy }}=\overline{\mathrm{y}}_{\text {sys }} \times \exp \left(\frac{\overline{\mathrm{X}}-\overline{\mathrm{x}}_{\text {sys }}}{\overline{\mathrm{X}}+\overline{\mathrm{x}}_{\text {sys }}}\right)$

and

$\hat{\bar{Y}}_{\text {Pesy }}=\overline{\mathrm{y}}_{\text {sys }} \times \exp \left(\frac{\overline{\mathrm{z}}_{\text {sys }}-\overline{\mathrm{z}}}{\overline{\mathrm{z}}_{\mathrm{sys}}+\overline{\mathrm{z}}}\right)$.

Variances of the ratio estimators $\quad \hat{\bar{Y}}_{\mathrm{R}}^{\text {sys }}, \hat{\overline{\mathrm{Y}}}_{\mathrm{P}}^{\text {sys }}, \quad \hat{\overline{\mathrm{Y}}}_{\text {Resy }}$ and $\quad \hat{\overline{\mathrm{Y}}}_{\text {Pesy }}$ are $\quad$ written $\quad$ as

$\mathrm{V}\left(\hat{\overline{\mathrm{Y}}}_{\mathrm{Rsys}}\right)=\frac{(\mathrm{N}-1)}{\mathrm{nN}} \overline{\mathrm{Y}}^{2}\left[\rho_{\mathrm{y}}^{*} \mathrm{C}_{\mathrm{y}}^{2}+\rho_{\mathrm{x}}^{*} \mathrm{C}_{\mathrm{x}}^{2}\left(1-2 \mathrm{~K} \sqrt{\rho^{* *}}\right)\right]$.

and

$\mathrm{V}\left(\hat{\overline{\mathrm{Y}}}_{\text {Resy }}\right)=\frac{(\mathrm{N}-1)}{\mathrm{nN}} \overline{\mathrm{Y}}^{2}\left[\rho_{\mathrm{y}}^{*} \mathrm{C}_{\mathrm{y}}^{2}+\rho_{\mathrm{x}}^{*}\left(\mathrm{C}_{\mathrm{x}}^{2} / 4\right)\left(1-4 \mathrm{~K} \sqrt{\rho^{* *}}\right)\right]$.

$\mathrm{V}\left(\hat{\overline{\mathrm{Y}}}_{\mathrm{Psys}}\right)=\frac{(\mathrm{N}-1)}{\mathrm{nN}} \overline{\mathrm{Y}}^{2}\left[\rho_{\mathrm{y}}^{*} \mathrm{c}_{\mathrm{y}}^{2}+\rho_{\mathrm{z}}^{*} \mathrm{c}_{\mathrm{z}}^{2}\left(1+2 \mathrm{k} \sqrt{\rho_{2}^{* *}}\right)\right]$.

$\mathrm{V}\left(\hat{\overline{\mathrm{Y}}}_{\text {Pesys }}\right)=\frac{(\mathrm{N}-1)}{\mathrm{nN}} \overline{\mathrm{Y}}^{2}\left[\rho_{\mathrm{y}}^{*} \mathrm{c}_{\mathrm{y}}^{2}+\rho_{\mathrm{z}}^{*}\left(\mathrm{c}_{\mathrm{z}}^{2} / 4\right)\left(1-4 \mathrm{k} \sqrt{\rho_{2}^{* *}}\right)\right]$.

where

$k=\rho_{\mathrm{yx}} \frac{\mathrm{C}_{\mathrm{y}}}{\mathrm{C}_{\mathrm{x}}}, \rho_{\mathrm{y}}^{*}=\left[1+\rho_{\mathrm{y}}(\mathrm{n}-1)\right], \quad \rho_{\mathrm{x}}^{*}=\left[1+\rho_{\mathrm{x}}(\mathrm{n}-1)\right], \quad \rho_{\mathrm{z}}^{*}=\left[1+\rho_{\mathrm{z}}(\mathrm{n}-1)\right]$,

$S_{y x}=\frac{1}{N-1} \sum_{i=1}^{K} \sum_{j=1}^{n}\left(x_{i j}-\bar{X}\right) \times\left(y_{i j}-\bar{Y}\right), \quad S_{x}^{2}=\frac{1}{N-1} \sum_{i=1}^{k} \sum_{j=1}^{n}\left(x_{i j}-\bar{X}\right)^{2}$,

$\mathrm{S}_{\mathrm{y}}^{2}=\frac{1}{\mathrm{~N}-1} \sum_{\mathrm{i}=1}^{\mathrm{k}} \sum_{\mathrm{j}=1}^{\mathrm{n}}\left(\mathrm{y}_{\mathrm{ij}}-\overline{\mathrm{Y}}\right)^{2}, \quad \mathrm{~S}_{\mathrm{z}}^{2}=\frac{1}{\mathrm{~N}-1} \sum_{\mathrm{i}=1}^{\mathrm{k}} \sum_{\mathrm{j}=1}^{\mathrm{n}}\left(\mathrm{z}_{\mathrm{ij}}-\overline{\mathrm{Z}}\right)^{2}$,

$\mathrm{k}^{*}=\rho_{\mathrm{yz}} \frac{\mathrm{C}_{\mathrm{y}}}{\mathrm{C}_{\mathrm{z}}} \quad$ and $\quad \rho_{2}^{* *}=\frac{\rho_{\mathrm{y}}^{*}}{\rho_{\mathrm{z}}^{*}}$.

and $\left(\rho_{y}, \rho_{x}, \rho_{z}\right)$ are the intra- class correlation coefficient between the units of a cluster corresponding the $(y, x, z)$ variates.

\section{SUGGESTED ESTIMATOR}

Singh (1967) envisaged ratio-cum -product estimator for population mean in simple random sampling. Tailor et al.(2011) studied properties of singh (1967) estimators in stratified random sampling. Singh et al.(2009) studied ratio -cum- product 
type exponential estimators. In simple random sampling assuming that the auxiliary variate $\mathrm{x}$ is positively correlated with the study variate $\mathrm{y}$ and the auxiliary variate $\mathrm{z}$ is negatively correlated with the $\mathrm{y}$, motivated by above cited work, the suggested raio-cum-product type exponential estimator for the population mean in systematic sampling is expressed as

$$
\hat{\bar{Y}}_{\mathrm{RPe}}^{\text {sys }}=\overline{\mathrm{y}}_{\mathrm{sys}} \exp \left(\frac{\overline{\mathrm{X}}-\overline{\mathrm{x}}_{\mathrm{sys}}}{\overline{\mathrm{X}}+\overline{\mathrm{x}}_{\mathrm{sys}}}\right) \exp \left(\frac{\overline{\mathrm{z}}_{\mathrm{sys}}-\overline{\mathrm{Z}}}{\overline{\mathrm{Z}}_{\mathrm{sys}}+\overline{\mathrm{Z}}}\right)
$$

To get essential property of $\hat{\overline{\mathrm{Y}}}_{\mathrm{RPe}}^{\text {sys }}$.i.e. bias and mean squared error ,it is assumed that

$\overline{\mathrm{y}}_{\text {sys }}=\overline{\mathrm{Y}}\left(1+\mathrm{e}_{0}\right), \overline{\mathrm{x}}_{\text {sys }}=\overline{\mathrm{X}}\left(1+\mathrm{e}_{1}\right), \overline{\mathrm{z}}_{\text {sys }}=\overline{\mathrm{Z}}\left(1+\mathrm{e}_{2}\right)$ such that

$\mathrm{E}\left(\mathrm{e}_{0}\right)=\mathrm{E}\left(\mathrm{e}_{1}\right)=\mathrm{E}\left(\mathrm{e}_{2}\right)=0$

$\mathrm{E}\left(\mathrm{e}_{0}^{2}\right)=\theta \mathrm{C}_{\mathrm{y}}^{2} \rho_{\mathrm{y}}^{*}, \quad \mathrm{E}\left(\mathrm{e}_{1}^{2}\right)=\theta \mathrm{C}_{\mathrm{x}}^{2} \rho_{\mathrm{x}}^{*}, \quad \mathrm{E}\left(\mathrm{e}_{2}^{2}\right)=\theta \mathrm{C}_{\mathrm{z}}^{2} \rho_{\mathrm{z}}^{*}$,

$\mathrm{E}\left(\mathrm{e}_{0} \mathrm{e}_{1}\right)=\theta \mathrm{k} \mathrm{C}_{\mathrm{x}}^{2} \sqrt{\rho_{\mathrm{y}}^{*}} \rho_{\mathrm{x}}^{*}, \mathrm{E}\left(\mathrm{e}_{0} \mathrm{e}_{2}\right)=\theta \mathrm{k}^{*} \mathrm{C}_{\mathrm{z}}^{2} \sqrt{\rho_{\mathrm{y}}^{*}} \rho_{\mathrm{z}}^{*}, \mathrm{E}\left(\mathrm{e}_{1} \mathrm{e}_{2}\right)=\theta \mathrm{k}^{* *} \mathrm{C}_{\mathrm{z}}^{2} \sqrt{\rho_{\mathrm{x}}^{*}} \rho_{\mathrm{z}}^{*}$

$C_{z}^{2}=\frac{S_{z}^{2}}{\bar{Z}^{2}}, \quad C_{x}^{2}=\frac{S_{x}^{2}}{\bar{X}^{2}}, \quad C_{y}^{2}=\frac{S_{y}^{2}}{\bar{Y}^{2}}, \quad \theta=\frac{N-1}{n N}, \rho_{y z}=\frac{S_{y z}}{S_{y} S_{z}}, \rho_{x z}=\frac{S_{x z}}{S_{x} S_{z}}$,

$\rho_{\mathrm{yx}}=\frac{\mathrm{S}_{\mathrm{yx}}}{\mathrm{S}_{\mathrm{y}} \mathrm{S}_{\mathrm{x}}}, \quad \rho_{\mathrm{x}}^{*}=\left(1+(\mathrm{n}-1) \rho_{\mathrm{x}}\right), \quad \rho_{\mathrm{y}}^{*}=\left(1+(\mathrm{n}-1) \rho_{\mathrm{y}}\right), \rho_{\mathrm{z}}^{*}=\left(1+(\mathrm{n}-1) \rho_{\mathrm{z}}\right)$

$\mathrm{k}^{* *}=\rho_{\mathrm{xz}}\left(\frac{\mathrm{c}_{\mathrm{x}}}{\mathrm{c}_{\mathrm{z}}}\right), \quad \mathrm{k}^{*}=\rho_{\mathrm{yz}}\left(\frac{\mathrm{c}_{\mathrm{y}}}{\mathrm{c}_{\mathrm{z}}}\right), \mathrm{k}=\rho_{\mathrm{yx}}\left(\frac{\mathrm{c}_{\mathrm{y}}}{\mathrm{c}_{\mathrm{x}}}\right)$,

Finally, upto the first degree of approximation, the bias and mean squared error of the suggested estimator are $\hat{\overline{\mathrm{Y}}}_{\mathrm{RPe}}^{\text {sys }}$ obtained as

$$
\mathrm{B}\left(\hat{\overline{\mathrm{Y}}}_{\mathrm{RPe}}^{\mathrm{sys}}\right)=\frac{(\mathrm{N}-1)}{\mathrm{nN}} \overline{\mathrm{Y}}^{2}\left[\frac{3}{8} \rho_{\mathrm{x}}^{*} \mathrm{c}_{\mathrm{x}}^{2}-\frac{1}{8} \rho_{\mathrm{z}}^{*} \mathrm{c}_{\mathrm{z}}^{2}+\frac{1}{2} \mathrm{k}^{*} \mathrm{c}_{\mathrm{z}}^{2} \sqrt{\rho_{\mathrm{y}}^{*}} \rho_{\mathrm{z}}^{*}-\frac{1}{2} \mathrm{kc}_{\mathrm{x}}^{2} \sqrt{\rho_{\mathrm{y}}^{*}} \rho_{\mathrm{x}}^{*}-\frac{1}{4} \mathrm{k}^{* *} \mathrm{c}_{\mathrm{z}}^{2} \sqrt{\rho_{\mathrm{x}}^{*} \rho_{\mathrm{z}}^{*}}\right]
$$

and

$$
\mathrm{V}\left(\hat{\bar{Y}}_{\mathrm{RPe}}^{\mathrm{sys}}\right)=\frac{(\mathrm{N}-1)}{\mathrm{nN}} \overline{\mathrm{Y}}^{2}\left[\rho_{\mathrm{y}}^{*} \mathrm{c}_{\mathrm{y}}^{2}+\rho_{\mathrm{x}}^{*}\left(\mathrm{c}_{\mathrm{x}}^{2} / 4\right)+\rho_{\mathrm{z}}^{*}\left(\mathrm{c}_{\mathrm{z}}^{2} / 4\right)+\mathrm{k}^{*} \mathrm{c}_{\mathrm{z}}^{2} \sqrt{\rho_{\mathrm{y}}^{*}} \rho_{\mathrm{z}}^{*}-\mathrm{kc}_{\mathrm{x}}^{2} \sqrt{\rho_{\mathrm{y}}^{*}} \rho_{\mathrm{x}}^{*}-\frac{1}{2} \mathrm{k}^{* *} \mathrm{c}_{\mathrm{z}}^{2} \sqrt{\rho_{\mathrm{x}}^{*} \rho_{\mathrm{z}}^{*}}\right]
$$

\section{EFFICIENCY COMPARISON}

Variance of classical-unbiased estimator for population mean is expressed as

$\mathrm{V}\left(\overline{\mathrm{y}}_{\mathrm{sys}}\right)=\frac{(\mathrm{N}-1)}{\mathrm{nN}} \overline{\mathrm{Y}}^{2} \rho_{\mathrm{y}}^{*} \mathrm{C}_{\mathrm{y}}^{2}$.

Comparision of (3.1), (1.7), (1.9), (1.11) and (2.3) show that the developed estimator $\hat{\bar{Y}}_{\mathrm{RPe}}^{\text {sys }} \quad$ would be more efficient than

(i) $\bar{y}_{\text {sys }}$ if

$$
\begin{aligned}
& \operatorname{Var}\left(\hat{\overline{\mathrm{Y}}}_{\mathrm{RPe}}^{\mathrm{sys}}\right)-\operatorname{Var}\left(\overline{\mathrm{y}}_{\mathrm{sys}}\right) \prec 0 \\
\Rightarrow & {\left[\frac{(\mathrm{N}-1)}{\mathrm{nN}} \overline{\mathrm{Y}}^{2}\left[\rho_{\mathrm{y}}^{*} \mathrm{c}_{\mathrm{y}}^{2}+\rho_{\mathrm{x}}^{*}\left(\mathrm{c}_{\mathrm{x}}^{2} / 4\right)+\rho_{\mathrm{z}}^{*}\left(\mathrm{c}_{\mathrm{z}}^{2} / 4\right)+\mathrm{k}^{*} \mathrm{c}_{\mathrm{z}}^{2} \sqrt{\rho_{\mathrm{y}}^{*}} \rho_{\mathrm{z}}^{*}-\mathrm{kc} \mathrm{x}_{\mathrm{x}}^{2} \sqrt{\rho_{\mathrm{y}}^{*}} \rho_{\mathrm{x}}^{*}-\frac{1}{2} \mathrm{k}^{* *} \mathrm{c}_{\mathrm{z}}^{2} \sqrt{\rho_{\mathrm{x}}^{*} \rho_{\mathrm{z}}^{*}}\right]-\frac{(\mathrm{N}-1)}{\mathrm{nN}} \overline{\mathrm{Y}}^{2} \rho_{\mathrm{y}}^{*} \mathrm{C}_{\mathrm{y}}^{2} .\right.}
\end{aligned}
$$


$\Rightarrow \frac{(\mathrm{N}-1)}{\mathrm{nN}} \overline{\mathrm{Y}}^{2}\left[\rho_{\mathrm{x}}^{*}\left(\mathrm{c}_{\mathrm{x}}^{2} / 4\right)+\rho_{\mathrm{z}}^{*}\left(\mathrm{c}_{\mathrm{z}}^{2} / 4\right)+\mathrm{k}^{*} \mathrm{c}_{\mathrm{z}}^{2} \sqrt{\rho_{\mathrm{y}}^{*}} \rho_{\mathrm{z}}^{*}-\mathrm{kc}_{\mathrm{x}}^{2} \sqrt{\rho_{\mathrm{y}}^{*}} \rho_{\mathrm{x}}^{*}-\frac{1}{2} \mathrm{k}^{* *} \mathrm{c}_{\mathrm{z}}^{2} \sqrt{\rho_{\mathrm{x}}^{*} \rho_{\mathrm{z}}^{*}}\right]<0$

(ii) $\hat{\bar{Y}}_{\text {Rsys }}$ if

$\operatorname{Var}\left(\hat{\overline{\mathrm{Y}}}_{\mathrm{RPe}}^{\text {sys }}\right)-\operatorname{Var}\left(\hat{\overline{\mathrm{Y}}}_{\mathrm{Rsys}}\right) \prec 0$,

$\Rightarrow\left[\frac{(\mathrm{N}-1)}{\mathrm{nN}} \overline{\mathrm{Y}}^{2}\left[\rho_{\mathrm{y}}^{*} \mathrm{c}_{\mathrm{y}}^{2}+\rho_{\mathrm{x}}^{*}\left(\mathrm{c}_{\mathrm{x}}^{2} / 4\right)+\rho_{\mathrm{z}}^{*}\left(\mathrm{c}_{\mathrm{z}}^{2} / 4\right)+\mathrm{k}^{*} \mathrm{c}_{\mathrm{z}}^{2} \sqrt{\rho_{\mathrm{y}}^{*}} \rho_{\mathrm{z}}^{*}-\mathrm{kc}_{\mathrm{x}}^{2} \sqrt{\rho_{\mathrm{y}}^{*}} \rho_{\mathrm{x}}^{*}-\frac{1}{2} \mathrm{k}^{* *} \mathrm{c}_{\mathrm{z}}^{2} \sqrt{\rho_{\mathrm{x}}^{*} \rho_{\mathrm{z}}^{*}}\right]-\right.$

$\frac{(\mathrm{N}-1)}{\mathrm{nN}} \overline{\mathrm{Y}}^{2}\left[\rho_{\mathrm{y}}^{*} \mathrm{C}_{\mathrm{y}}^{2}+\rho_{\mathrm{x}}^{*} \mathrm{C}_{\mathrm{x}}^{2}\left(1-2 \mathrm{~K} \sqrt{\rho^{* *}}\right)\right] \prec 0$

$\Rightarrow\left[\frac{(\mathrm{N}-1)}{\mathrm{nN}} \overline{\mathrm{Y}}^{2}\left[\rho_{\mathrm{x}}^{*}\left(\mathrm{c}_{\mathrm{x}}^{2} / 4\right)-\rho_{\mathrm{x}}^{*} \mathrm{C}_{\mathrm{x}}^{2}\left(1-2 \mathrm{~K} \sqrt{\rho^{* *}}\right)+\rho_{\mathrm{z}}^{*}\left(\mathrm{c}_{\mathrm{z}}^{2} / 4\right)+\mathrm{k}^{*} \mathrm{c}_{\mathrm{z}}^{2} \sqrt{\rho_{\mathrm{y}}^{*}} \rho_{\mathrm{z}}^{*}-\mathrm{kc}_{\mathrm{x}}^{2} \sqrt{\rho_{\mathrm{y}}^{*}} \rho_{\mathrm{x}}^{*}-\frac{1}{2} \mathrm{k}^{* *} \mathrm{c}_{\mathrm{z}}^{2} \sqrt{\rho_{\mathrm{x}}^{*} \rho_{\mathrm{z}}^{*}}\right] \prec 0\right.$

(iii) $\hat{\bar{Y}}_{\text {Resys }}$ if

$\operatorname{Var}\left(\hat{\bar{Y}}_{\mathrm{RPe}}^{\text {sys }}\right)-\operatorname{Var}\left(\hat{\overline{\mathrm{Y}}}_{\text {Resys }}\right) \prec 0$,

$\Rightarrow\left[\frac{(\mathrm{N}-1)}{\mathrm{nN}} \overline{\mathrm{Y}}^{2}\left[\rho_{\mathrm{y}}^{*} \mathrm{c}_{\mathrm{y}}^{2}+\rho_{\mathrm{x}}^{*}\left(\mathrm{c}_{\mathrm{x}}^{2} / 4\right)+\rho_{\mathrm{z}}^{*}\left(\mathrm{c}_{\mathrm{z}}^{2} / 4\right)+\mathrm{k}^{*} \mathrm{c}_{\mathrm{z}}^{2} \sqrt{\rho_{\mathrm{y}}^{*}} \rho_{\mathrm{z}}^{*}-\mathrm{kc}_{\mathrm{x}}^{2} \sqrt{\rho_{\mathrm{y}}^{*}} \rho_{\mathrm{x}}^{*}-\frac{1}{2} \mathrm{k}^{* *} \mathrm{c}_{\mathrm{z}}^{2} \sqrt{\rho_{\mathrm{x}}^{*} \rho_{\mathrm{z}}^{*}}\right]-\right.$

$\frac{(\mathrm{N}-1)}{\mathrm{nN}} \overline{\mathrm{Y}}^{2}\left[\rho_{\mathrm{y}}^{*} \mathrm{C}_{\mathrm{y}}^{2}+\rho_{\mathrm{x}}^{*}\left(\mathrm{C}_{\mathrm{x}}^{2} / 4\right)\left(1-4 \mathrm{~K} \sqrt{\rho^{* *}}\right)\right] \prec 0$

$\Rightarrow\left[\frac{(\mathrm{N}-1)}{\mathrm{nN}} \overline{\mathrm{Y}}^{2}\left[\rho_{\mathrm{x}}^{*} \mathrm{c}_{\mathrm{x}}^{2} \mathrm{k} \sqrt{\rho^{* *}}+\rho_{\mathrm{z}}^{*}\left(\mathrm{c}_{\mathrm{z}}^{2} / 4\right)+\mathrm{k}^{*} \mathrm{c}_{\mathrm{z}}^{2} \sqrt{\rho_{\mathrm{y}}^{*}} \rho_{\mathrm{z}}^{*}-\mathrm{k} \mathrm{c}_{\mathrm{x}}^{2} \sqrt{\rho_{\mathrm{y}}^{*}} \rho_{\mathrm{x}}^{*}-\frac{1}{2} \mathrm{k}^{* *} \mathrm{c}_{\mathrm{z}}^{2} \sqrt{\rho_{\mathrm{x}}^{*} \rho_{\mathrm{z}}^{*}}\right] \prec 0\right.$ (4.3.2)

(iv) $\hat{\bar{Y}}_{\text {Psys }}$ if

$\operatorname{Var}\left(\hat{\overline{\mathrm{Y}}}_{\mathrm{RPe}}^{\text {sys }}\right)-\operatorname{Var}\left(\hat{\overline{\mathrm{Y}}}_{\mathrm{Psys}}\right) \prec 0$,

$\Rightarrow\left[\frac{(\mathrm{N}-1)}{\mathrm{nN}} \overline{\mathrm{Y}}^{2}\left[\rho_{\mathrm{y}}^{*} \mathrm{c}_{\mathrm{y}}^{2}+\rho_{\mathrm{x}}^{*}\left(\mathrm{c}_{\mathrm{x}}^{2} / 4\right)+\rho_{\mathrm{z}}^{*}\left(\mathrm{c}_{\mathrm{z}}^{2} / 4\right)+\mathrm{k}^{*} \mathrm{c}_{\mathrm{z}}^{2} \sqrt{\rho_{\mathrm{y}}^{*}} \rho_{\mathrm{z}}^{*}-\mathrm{kc} \mathrm{c}_{\mathrm{x}}^{2} \sqrt{\rho_{\mathrm{y}}^{*}} \rho_{\mathrm{x}}^{*}-\frac{1}{2} \mathrm{k}^{* *} \mathrm{c}_{\mathrm{z}}^{2} \sqrt{\rho_{\mathrm{x}}^{*} \rho_{\mathrm{z}}^{*}}\right]-\right.$

$\frac{(\mathrm{N}-1)}{\mathrm{nN}} \overline{\mathrm{Y}}^{2}\left[\rho_{\mathrm{y}}^{*} \mathrm{c}_{\mathrm{y}}^{2}+\rho_{\mathrm{z}}^{*} \mathrm{c}_{\mathrm{z}}^{2}\left(1+2 \mathrm{k} \sqrt{\rho_{2}^{* *}}\right)\right] \prec 0$

$\Rightarrow \frac{(\mathrm{N}-1)}{\mathrm{nN}} \overline{\mathrm{Y}}^{2}\left[\rho_{\mathrm{x}}^{*}\left(\mathrm{c}_{\mathrm{x}}^{2} / 4\right)+\rho_{\mathrm{z}}^{*}\left(\mathrm{c}_{\mathrm{z}}^{2} / 4\right)-\rho_{\mathrm{z}}^{*} \mathrm{c}_{\mathrm{z}}^{2}\left(1+2 \mathrm{k} \sqrt{\rho_{2}^{* *}}\right)+\mathrm{k}^{*} \mathrm{c}_{\mathrm{z}}^{2} \sqrt{\rho_{\mathrm{y}}^{*}} \rho_{\mathrm{z}}^{*}-\mathrm{kc}_{\mathrm{x}}^{2} \sqrt{\rho_{\mathrm{y}}^{*}} \rho_{\mathrm{x}}^{*}-\frac{1}{2} \mathrm{k}^{* *} \mathrm{c}_{\mathrm{z}}^{2} \sqrt{\rho_{\mathrm{x}}^{*} \rho_{\mathrm{z}}^{*}}\right] \prec 0(4.3 .3)$

(v) $\hat{\bar{Y}}_{\text {Pesys }}$ if

$\operatorname{Var}\left(\overline{\mathrm{Y}}_{\mathrm{RPe}}^{\text {sys }}\right)-\operatorname{Var}\left(\overline{\mathrm{Y}}_{\text {Pesys }}\right) \prec 0$,

$\Rightarrow \frac{(\mathrm{N}-1)}{\mathrm{nN}} \overline{\mathrm{Y}}^{2}\left[\rho_{\mathrm{y}}^{*} \mathrm{c}_{\mathrm{y}}^{2}+\rho_{\mathrm{x}}^{*}\left(\mathrm{c}_{\mathrm{x}}^{2} / 4\right)+\rho_{\mathrm{z}}^{*}\left(\mathrm{c}_{\mathrm{z}}^{2} / 4\right)+\mathrm{k}^{*} \mathrm{c}_{\mathrm{z}}^{2} \sqrt{\rho_{\mathrm{y}}^{*}} \rho_{\mathrm{z}}^{*}-\mathrm{k} \mathrm{c}_{\mathrm{x}}^{2} \sqrt{\rho_{\mathrm{y}}^{*}} \rho_{\mathrm{x}}^{*}-\frac{1}{2} \mathrm{k}^{* *} \mathrm{c}_{\mathrm{z}}^{2} \sqrt{\rho_{\mathrm{x}}^{*} \rho_{\mathrm{z}}^{*}}\right]-$

$\frac{(\mathrm{N}-1)}{\mathrm{nN}} \overline{\mathrm{Y}}^{2}\left[\rho_{\mathrm{y}}^{*} \mathrm{c}_{\mathrm{y}}^{2}+\rho_{\mathrm{z}}^{*}\left(\mathrm{c}_{\mathrm{z}}^{2} / 4\right)\left(1-4 \mathrm{k} \sqrt{\rho_{2}^{* *}}\right)\right] \prec 0$ 


$$
\begin{aligned}
& \Rightarrow \frac{(\mathrm{N}-1)}{\mathrm{nN}} \overline{\mathrm{Y}}^{2}\left[\rho_{\mathrm{x}}^{*}\left(\mathrm{c}_{\mathrm{x}}^{2} / 4\right)+\rho_{\mathrm{z}}^{*} \mathrm{c}_{\mathrm{z}}^{2} \mathrm{k} \sqrt{\rho_{2}^{* *}}+\mathrm{k}^{*} \mathrm{c}_{\mathrm{z}}^{2} \sqrt{\rho_{\mathrm{y}}^{*}} \rho_{\mathrm{z}}^{*}-\mathrm{kc} \mathrm{x}_{\mathrm{x}}^{2} \sqrt{\rho_{\mathrm{y}}^{*}} \rho_{\mathrm{x}}^{*}-\frac{1}{2} \mathrm{k}^{* *} \mathrm{c}_{\mathrm{z}}^{2} \sqrt{\rho_{\mathrm{x}}^{*} \rho_{\mathrm{z}}^{*}}\right] \\
& \Rightarrow \frac{(\mathrm{N}-1)}{\mathrm{nN}} \overline{\mathrm{Y}}^{2}\left[\rho_{\mathrm{x}}^{*}\left(\mathrm{c}_{\mathrm{x}}^{2} / 4\right)+\rho_{\mathrm{z}}^{*} \mathrm{c}_{\mathrm{z}}^{2} \mathrm{k} \sqrt{\rho^{* *}}+\mathrm{k}^{*} \mathrm{c}_{\mathrm{z}}^{2} \sqrt{\rho_{\mathrm{y}}^{*}} \rho_{\mathrm{z}}^{*}-\mathrm{kc}_{\mathrm{x}}^{2} \sqrt{\rho_{\mathrm{y}}^{*}} \rho_{\mathrm{x}}^{*}-\frac{1}{2} \mathrm{k}^{* *} \mathrm{c}_{\mathrm{z}}^{2} \sqrt{\rho_{\mathrm{x}}^{*} \rho_{\mathrm{z}}^{*}}\right] \prec 0(4.3 .4)
\end{aligned}
$$

\section{EMPIRICAL STUDY}

This section compares considerd estimators empirically. For this purpose an artificial population is being considered. Parameters of this artificial population are given below.

$\overline{\mathrm{X}}=44.47, \overline{\mathrm{Y}}=80, \overline{\mathrm{Z}}=44.47, \mathrm{C}_{\mathrm{x}}=0.28, \mathrm{C}_{\mathrm{y}}=0.56, \mathrm{C}_{\mathrm{z}}=0.43, \mathrm{~S}_{\mathrm{x}}^{2}=149.55, \mathrm{~S}_{\mathrm{y}}^{2}=2000$,

$S_{z}^{2}=427.83, \quad S_{x z}=-241.06, \quad S_{y z}=-902.86, \quad S_{x y}=-241.06, \quad \rho_{x y}=0.9848, \quad \rho_{y z}=-0.9760$ $\rho_{\mathrm{zx}}=-0.9530, \rho_{\mathrm{x}}=0.707, \rho_{\mathrm{y}}=0.6652, \rho_{\mathrm{z}}=0.5487, \mathrm{~N}=15, \mathrm{n}=3$

Table (4.1) Percent Relative Efficiencies of $\hat{\bar{Y}}_{\text {Rsys }}, \hat{\bar{Y}}_{\text {Psys }}, \hat{\bar{Y}}_{\text {Resys }}, \hat{\bar{Y}}_{\text {Pesys }}$ and $\hat{\bar{Y}}_{\text {RPesy }}^{\text {sys }}$ with respect to $\overline{\mathrm{y}}_{\text {sys }}$.

\begin{tabular}{|c|c|}
\hline Estimator & PRE(., $\left.\overline{\mathrm{y}}_{\text {sys }}\right)$ \\
\hline$\overline{\mathrm{y}}_{\text {sys }}$ & 100.00 \\
\hline$\hat{\overline{\mathrm{Y}}}_{\text {Rsys }}$ & 389.620 \\
\hline$\hat{\overline{\mathrm{Y}}}_{\text {Psys }}$ & 189.452 \\
\hline$\hat{\overline{\mathrm{Y}}}_{\text {Resys }}$ & 177.434 \\
\hline$\hat{\overline{\mathrm{Y}}}_{\text {pesys }}$ & 139.318 \\
\hline$\hat{\overline{\mathrm{Y}}}_{\text {RPe }}^{\text {sys }}$ & 617.606 \\
\hline
\end{tabular}

Table 4.2 Empirical Exhibition of Theoretical Conditions Obtained in Section-(4.3)

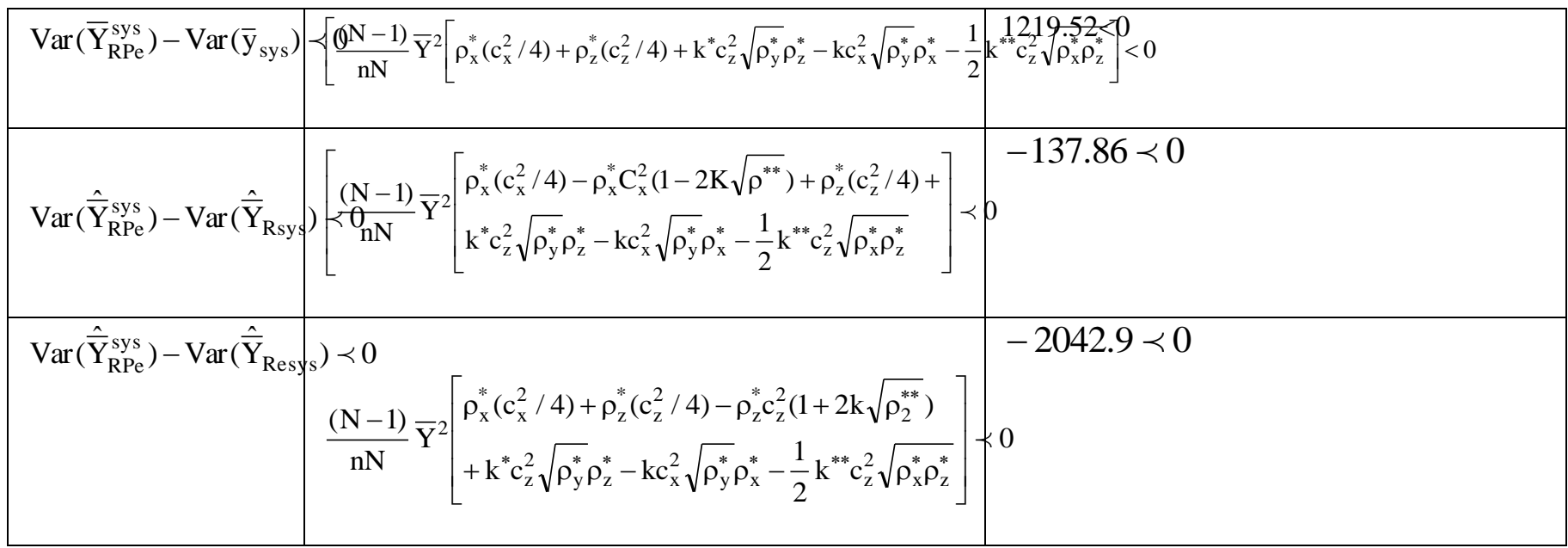




\begin{tabular}{|c|c|c|c|}
\hline $\operatorname{Var}\left(\hat{\overline{\mathrm{Y}}}_{\mathrm{RPe}}^{\text {sys }}\right)-\operatorname{Var}\left(\hat{\overline{\mathrm{Y}}}_{\mathrm{Psys}}\right.$ & $\frac{\left.\gamma^{Q}-1\right)}{\mathrm{nN}} \overline{\mathrm{Y}}^{2}[$ & {$\left[\begin{array}{l}\rho_{\mathrm{x}}^{*}\left(\mathrm{c}_{\mathrm{x}}^{2} / 4\right)+\rho_{\mathrm{z}}^{*}\left(\mathrm{c}_{\mathrm{z}}^{2} / 4\right)-\rho_{\mathrm{z}}^{*} \mathrm{c}_{\mathrm{z}}^{2}\left(1+2 \mathrm{k} \sqrt{\rho_{\mathrm{z}}^{* *}}\right) \\
+\mathrm{k}^{*} \mathrm{c}_{\mathrm{z}}^{2} \sqrt{\rho_{\mathrm{y}}^{*}} \rho_{\mathrm{z}}^{*}-\mathrm{kc}_{\mathrm{x}}^{2} \sqrt{\rho_{\mathrm{y}}^{*}} \rho_{\mathrm{x}}^{*}-\frac{1}{2} \mathrm{k}^{* *} \mathrm{c}_{\mathrm{z}}^{2} \sqrt{\rho_{\mathrm{x}}^{*} \rho_{\mathrm{z}}^{*}}\end{array}\right.$} & Ł0 $-5197.93 \prec 0$ \\
\hline $\operatorname{Var}\left(\hat{\overline{\mathrm{Y}}}_{\mathrm{RPe}}^{\text {sys }}\right)-\operatorname{Var}\left(\hat{\overline{\mathrm{Y}}}_{\text {Pesy }}\right.$ & $\frac{(\mathrm{N}-1)}{\mathrm{nN}} \overline{\mathrm{Y}}^{2}$ & {$\left[\begin{array}{l}\rho_{\mathrm{x}}^{*}\left(\mathrm{c}_{\mathrm{x}}^{2} / 4\right)+\rho_{\mathrm{z}}^{*} \mathrm{c}_{\mathrm{z}}^{2} \mathrm{k} \sqrt{\rho^{* *}}+\mathrm{k}^{*} \mathrm{c}_{\mathrm{z}}^{2} \sqrt{\rho_{\mathrm{y}}^{*}} \rho_{\mathrm{z}}^{*} \\
-\mathrm{k} \mathrm{c}_{\mathrm{x}}^{2} \sqrt{\rho_{\mathrm{y}}^{*}} \rho_{\mathrm{x}}^{*}-\frac{1}{2} \mathrm{k}^{* *} \mathrm{c}_{\mathrm{z}}^{2} \sqrt{\rho_{\mathrm{x}}^{*} \rho_{\mathrm{z}}^{*}}\end{array}\right]$} & $\prec 0-2646.2<0$ \\
\hline
\end{tabular}

\section{CONCLUSIONS}

In this paper, a ratio-cum-product type exponential estimator for population mean has been developed using exponential function. Theoretical comparsion has been done in section 3 where conditions have been obtained under which developed estimator has less mean squared error as compared to other considered estimators. Table 4.1 shows that the developed ratiocum-product estimator has maximum PRE among all other considered estimators. In addition ,Table 4.2 exhibits that theoretical conditions are satisfied for the given empirical illustration. Thus it can be conclude that estimator developed in this work is more efficient then $\overline{\mathrm{y}}_{\text {sys }} \hat{\overline{\mathrm{Y}}}_{\text {Rsys }}, \hat{\overline{\mathrm{Y}}}_{\text {Psys }}, \hat{\overline{\mathrm{Y}}}_{\text {Resys }}, \hat{\overline{\mathrm{Y}}}_{\text {Pesys }}$. and recommended for estimation of population mean if conditions obtained in section 3 are satisfied.

\section{REFERENCES}

[1]. Bahl, S. and Tuteja, R.K. (1991). Ratio and product type exponential estimator. Info. \& Opti. Sci. $\quad$ XIII, $\quad 159-\quad 163$.

[2]. Shukla, N. D. (1971). Systematic sampling and product method of estimation. Proceedings of all India Seminar on Demography and Statistics. B.H.U., Varanasi, India.

[3]. Singh, M. P. (1967). Ratio-cum-product method of estimation. Metrika, 12, 34- 42.

[4]. Singh, H. P. and Singh, R. (1998). Almost unbiased ratio and product type estimators in systematic sampling. Questiio, 22,3, 403-416.

[5]. Singh, H. P., Tailor, R. and Jatwa, N. K. (2011). Modified ratio and product estimators for population mean in systematic sampling. J. Mod. Appl. Statist. Meth. 10 , 2,424-435.

[6]. Swain, A. K. P. C. (1964). The use of systematic sampling ratio estimate. J. Ind. Statist. Assoc., $\quad 2,160-\quad 164$. 